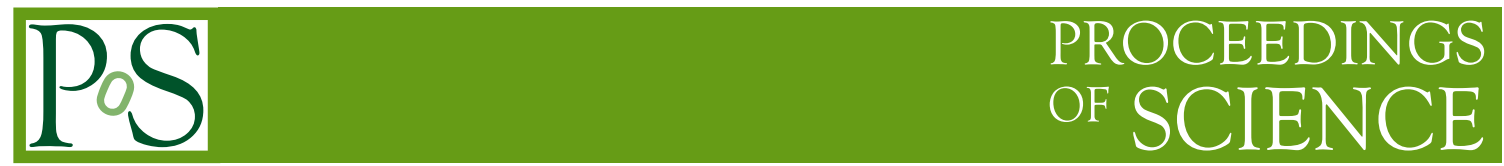

\title{
New results from COMPASS
}

\author{
Eva-Maria Kabuß ${ }^{* \dagger}$ \\ Institut für Kernphysik, Universität Mainz, D55099 Mainz \\ - on behalf of the COMPASS collaboration - \\ E-mail: emkekph.uni-mainz.de
}

An overview on recent COMPASS results is given, including the extraction of the longitudinal spin structure functions interpreted with a NLO QCD fit, new results on the gluon polarisation and a measurement of pion and kaon multiplicities with a LO extraction of quark-to-hadron fragmentation functions.

The European Physical Society Conference on High Energy Physics 22-29 July 2015

Vienna, Austria

\footnotetext{
*Speaker.

$\dagger$ supported by the BMBF
} 


\section{Introduction}

Since the surprising observation by the EMC [1] that only a small part of the proton spin is carried by quarks, a lot of experimental and theoretical studies were performed that shed more light onto the so-called spin puzzle, see e.g. Ref. [2]. All the new experiments show that only about $30 \%$ of the nucleon spin is carried by quarks. Other contributions stem from gluon spins and orbital angular momenta of quarks and gluons. The contribution of the different quarks flavours to the nucleon spin is studied using asymmtries of identified hadrons. Here, in addition to the well known spin-averaged quark distributions the fragmentation functions of quarks into hadrons are needed as inputs to the analysis. But especially the information on the strange quark fragmentation is scarce. Thus, COMPASS is also extracting fragmentation functions from the multiplicities of identified hadrons.

In the COMPASS experiment at CERN the spin puzzle has been studied using inclusive and semi-inclusive measurements in the scattering of polarised leptons off polarised nucleons. In the following, new results are summarised that contribute to the measurement of the contribution of the different quark flavours and gluons to the nucleon spin.

\section{COMPASS experiment}

The COMPASS experiment [3] is situated in the north area of the CERN SPS at the unique M2 muon beamline. It delivers a highly polarised beam of $\mu^{+}$or $\mu^{-}$with energies between 100 and $200 \mathrm{GeV}$. Most of the COMPASS data taking with muons was done at $160 \mathrm{GeV}$, except for 2011 when $200 \mathrm{GeV}$ was used.

The heart of the experiment is the solid state polarised target housed in a large aperture superconducting solenoid with a field of $2.5 \mathrm{~T}$. The target material is polarised using dynamic nuclear polarisation and is cooled below $100 \mathrm{mK}$ with the help of a ${ }^{3} \mathrm{He} /{ }^{4} \mathrm{He}$ dilution refrigerator. The target consists of two or three cells filled with ${ }^{6} \mathrm{LiD}$ (up to 2006) or $\mathrm{NH}_{3}$ (from 2007 onwards) polarised in opposite directions. These directions were reversed in regular intervals in order to minimise acceptance effects in the determination of asymmetries. A polarisation of about $90 \%$ is obtained for $\mathrm{NH}_{3}$, used as a proton target, and of about $50 \%$ for ${ }^{6} \mathrm{LiD}$, used as a deuteron target.

The spectrometer consists of a beam spectrometer in front of the target and a large acceptance double stage open dipole spectrometer behind the target. In the first stage tracks with low momenta and large angles are detected, while in the second stage fast forward going particles including the scattered muons are measured. Muons are identified by their passage though concrete or iron absorbers, while particle identification for hadrons is provided by a ring imaging Cherenkov detector (RICH) in the first stage that allows for $\pi / \mathrm{K} / \mathrm{p}$ separation.

The analysis is done in terms of the following kinematic variables: the negative square of the four momentum transfer $Q^{2}$, the relative energy of the virtual photon $y$, the Bjorken variable $x=Q^{2} / 2 M y E$ with $M$ the nucleon mass and $E$ the energy of the incoming muon in the lab system. The deep inelastic scattering regime is selected by requiring $Q^{2}>1(\mathrm{GeV} / c)^{2}$ and $0.1<y<0.9$, which implies $0.0025(0.004)<x<0.7$ for $200 \mathrm{GeV}(160 \mathrm{GeV})$ incicent muon energy and for the invariant mass of the final state $W^{2}>12\left(\mathrm{GeV} / c^{2}\right)^{2}$. 


\section{Spin structure functions}

Using events with reconstructed incoming and scattered muons attached to an interaction vertex the longitudinal virtual-photon asymmetries $A_{1}$ are determined in bins of $x$ and $Q^{2}$ from the raw asymmetries. Corrections are applied for target and beam polarisations, the dilution factor giving the amount of polarisable nucleons in the target material and the depolarisation factor describing the polarisation transfer from the lepton to the virtual photon [4-6]. At this stage also polarised and polarisation averaged radiative corrections are applied. The systematic uncertainties of the measurements stem from the above mentioned correction factors and possible false asymmetries. From the extracted asymmetries the spin structure function $g_{1}$ is determined using $g_{1}\left(x, Q^{2}\right)=A_{1}\left(x, Q^{2}\right) \cdot F_{2}\left(x, Q^{2}\right) /\left[2 x\left(1+R\left(x, Q^{2}\right)\right)\right]$. Here, $F_{2}$ is the spin averaged nucleon structure functions and $R=\sigma_{L} / \sigma_{T}$ is the ratio of the absorption cross section for longitudinal and transverse virtual photons. The new results obtained at $200 \mathrm{GeV}$ incident muon energy compare well with previous measurements and extend the kinematic range towards low $x$ and large $Q^{2}$ [6].

All world data on $g_{1}^{\mathrm{p}}, g_{1}^{\mathrm{d}}$ and $g_{1}^{\mathrm{n}}$ were used in a next-to-leading order (NLO) QCD fit to extract the polarised parton distributions (PDF) [6]. For this fit, a cut $W^{2}>10\left(\mathrm{GeV} / c^{2}\right)^{2}$ is applied to all data sets to reduce the influence of higher order effects, similar to Ref. [7]. The description of the data is very good as illustrated for the COMPASS data in Fig. 1. In this fit also the first
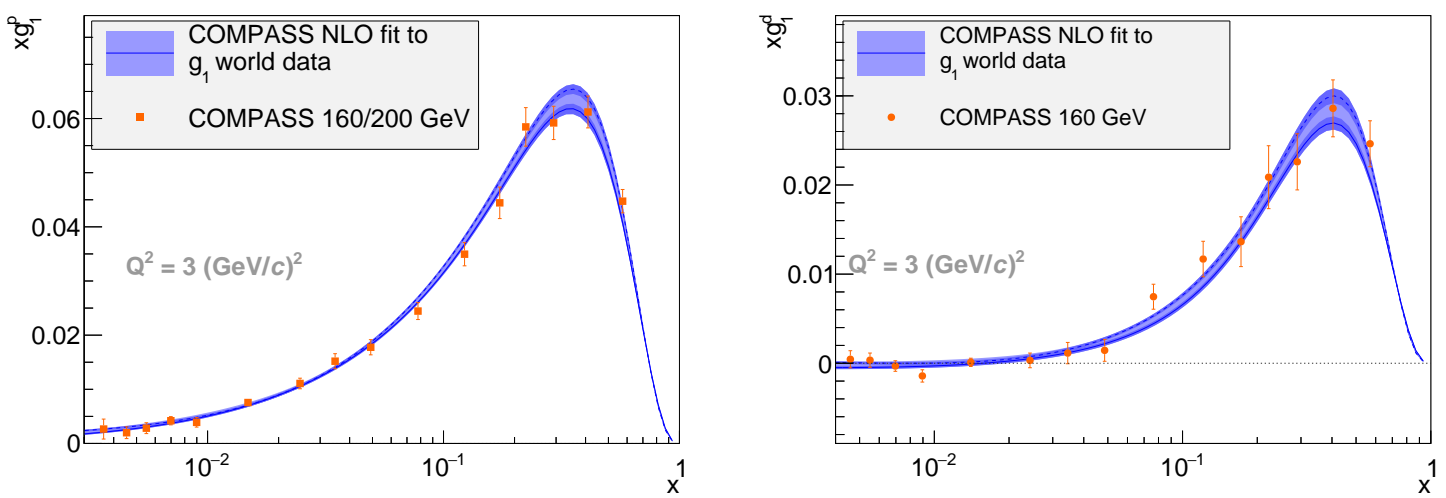

Figure 1: Left: Results for $x g_{1}^{\mathrm{p}}$ combining the new $200 \mathrm{GeV}$ data [6] with the one taken at $160 \mathrm{GeV}$ [5], right: results for $x g_{1}^{\mathrm{d}}$ from [4]. All data are evolved to $Q^{2}=3(\mathrm{GeV} / c)^{2}$ and compared to the new COMPASS NLO QCD fit. Dark bands represent the statistical, light bands the full uncertainty of the fit (for details see Ref. [6]).

moments of the PDFs are obtained. The result for the contribution of all quarks to the nucleon spin of $0.26<\Delta \Sigma<0.36$ is consistent with previous analyses. The larger systematic uncertainty in the COMPASS analysis compared toprevious ones is due to a very detailed study of the influence of the functional form assumed at the starting scale of $Q_{0}^{2}=1(\mathrm{GeV} / c)^{2}$. Together with the limited $Q^{2}$ range of the world data this leads to a very large uncertainty on the gluon polarisation, so that the sign of its first moment cannot be determined using the inclusive data only.

\section{Gluon polarisation}

To gain sensitivity to the gluon polarisation other processes, e.g. photon gluon fusion (PGF), 
that are directly sensitive to the gluon polarisation, can be employed. In leading order QCD the measured PGF asymmetry is related to the product of the analysing power of the process and the gluon polarisation $\Delta g$. In COMPASS two different channels are used to extract the gluon polarisation. In the first analysis, the PGF process is signalled by open charm production using identified kaons and pions from $\mathrm{D}$ meson decays. As the decay vertices of the $\mathrm{D}$ mesons cannot be observed in a fixed target experiment with a large solid state target, the subtraction of the combinatorial background relies on fits of the invariant mass distributions. The resulting asymmetries are analysed in LO and NLO QCD [8]. Albeit the fact that the theoretical uncertainties are small, this channel is hampered by larger statistical uncertainties due to the high combinatorial background. The second analysis makes use of the production of light quarks that acquire large transverse momenta in the PGF process. This dependence on the transverse momentum helps to disentangle the contributions from different processes to the transverse momentum dependence. The benefit is a large data sample, although the background due to the leading process and QCD Compton scattering has to be subtracted using Monte Carlo simulations. In a re-analysis [9], superseding the results published in [10], a value of $\langle\Delta g / g\rangle^{\mathrm{LO}}=0.11 \pm 0.038$ (stat) \pm 0.035 (syst) is obtained at a scale of $3(\mathrm{GeV} / c)^{2}$ and at an average gluon momentum fraction $x_{\mathrm{g}} \approx 0.1$. This is the most precise direct measurement of the gluon polarisation and clearly indicates that the gluon polarisation is positive in the measured range, consistent with a QCD fit that includes recent results from polarised proton-proton collisions at RHIC [7,11]. The large data sample permits to extact the results in three bins of $x_{\mathrm{g}}$. Figure 2 shows a comparison of the existing direct measurements of the gluon polarisation in LO QCD.
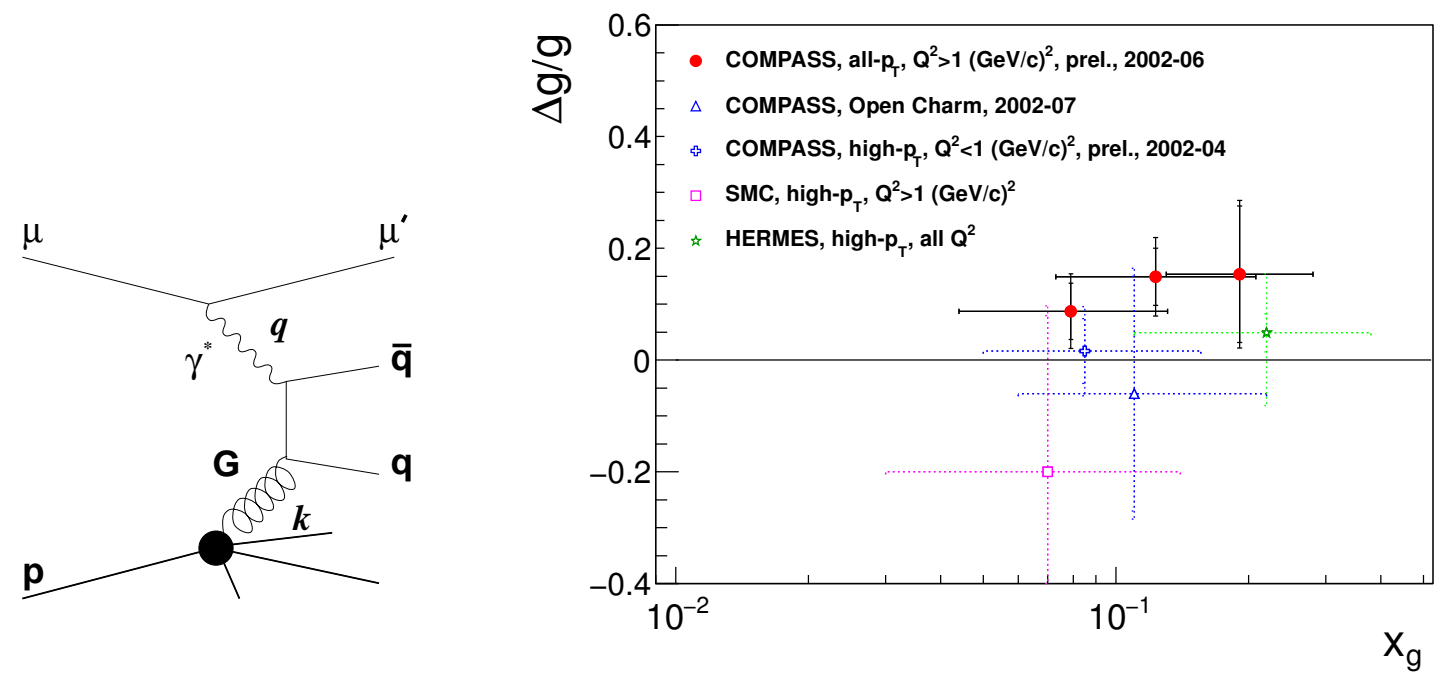

Figure 2: Left: The photon-gluon fusion process in DIS, right: comparison of results for direct measurements of the gluon polarisation including the three preliminary new COMPASS points.

\section{Multiplicities and fragmentation functions}

With the help of semi-inclusive asymmetries of identified hadrons also flavour separated quark polarisations can be studied. In such an analysis other inputs like spin averaged structure functions 
and quark-to-hadron fragmentation functions are needed. As shown in Ref. [12], the current knowledge on especially the strange quark fragmentation functions does not allow to draw conclusions on the strange quark polarisation, while the light quark polarisations are well measured. Thus an improved determination of fragmentation functions is mandatory to enable a reliable interpretation of the measured hadron asymmetries. With part of the data measured with the isoscalar ${ }^{6} \mathrm{LiD}$ target COMPASS embarked on the determination of identified hadrons multiplicities that are related in LO QCD to the fragmentation functions $D_{\mathrm{q}}^{\mathrm{h}}$ via

$$
M_{\mathrm{h}}\left(x, Q^{2}, z\right)=\frac{1}{N_{\mathrm{DIS}}\left(x, Q^{2}\right)} \frac{\mathrm{d} N_{\mathrm{h}}\left(x, Q^{2}, z\right)}{\mathrm{d} z}=\frac{\sum_{q} e_{q}^{2} q\left(x, Q^{2}\right) D_{q}^{\mathrm{h}}\left(z, Q^{2}\right)}{\sum_{q} e_{q}^{2} q\left(x, Q^{2}\right)} .
$$

Results are obtained for charged pion and kaon multiplicities in 3-dimensional bins of $x, y$ and $z$. In contrast to the above discussed asymmetry determinations these measurements require a detailed evaluation of the COMPASS acceptance including efficiencies and smearing effects, while the probabilities for particle identification were determined from data using two body decays of $\lambda$, $\phi$ and $\mathrm{K}^{0}$. The obtained results are illustrated in Fig. 3 for positive kaons [13].

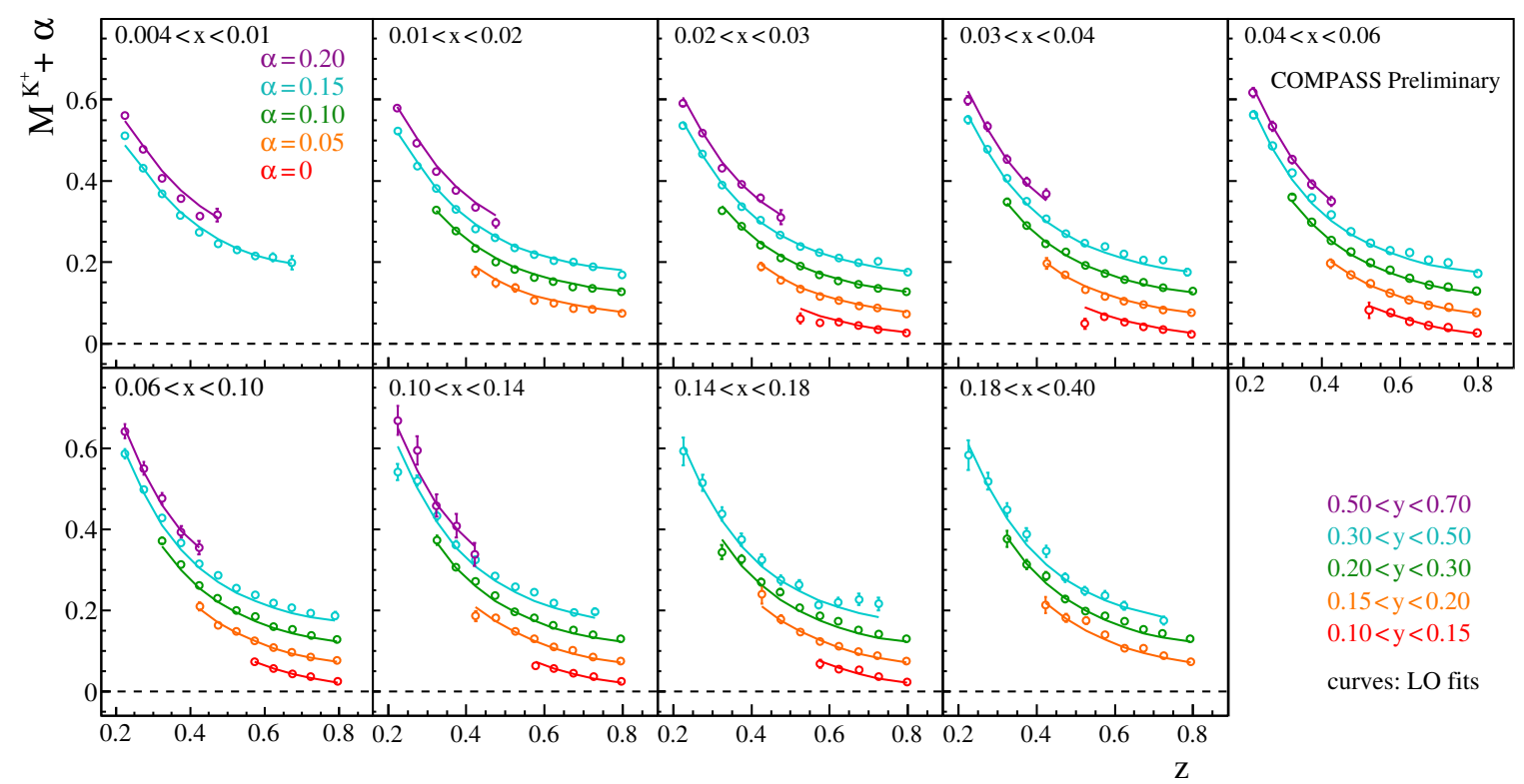

Figure 3: Positive kaon multiplicities versus $z$ for $8 x$ bins and $5 y$ bins (staggered for clarity) with statistical uncertainties. The curves correspond the COMPASS LO QCD fit of $\mathrm{K}^{+}$and $\mathrm{K}^{-}$multiplicities.

Using the preliminary $\mathrm{K}^{+} / \mathrm{K}^{-}$and $\pi^{+} / \pi^{-}$results in LO QCD fits, first results for the favoured and unfavoured fragmentation functions were obtained using the code of Ref. [14]. For pions they agree well with recent global extractions in NLO QCD [15-17], while for kaons the light quark fragmentation functions are much larger than previously estimated. The extraction of the strange quark fragmentation function is ongoing, also the determination of neutral kaon multiplicities.

\section{Conclusions}

New results from COMPASS for the quark and gluon polarisations were presented as well as a 
study of charged pion and kaon multiplicities leading to the evaluation of fragmentation fucntions. The latter were obtained using an isoscalar solid state target. These studies will be continued using data obtained in 2012 with a liquid hydrogen target, where secondary interactions of the produced hadrons are of much less importance allowing for a reduction of the systematic uncertainties in the multiplicity determination and thus in the extracted fragmentation functions. Moreover, the measurement of deeply virtual Compton scattering that will be performed in 2016/7 after the short exploratory run in 2012 will help to shed light on the still unknown contribution of quark orbital angular momenta to the nucleon spin.

\section{References}

[1] EM Collaboration, J. Ashman et al., Phys. Lett. B 206 (1988) 364; Nucl. Phys. B 328 (1989) 1.

[2] C.A. Aidala, S.D. Bass, D. Hasch and G.K. Mallot, Rev. Mod. Phys. 85 (2013) 655.

[3] COMPASS Collaboration, P. Abbon et al., Nucl. Instr. Meth. A 577 (2007) 455.

[4] COMPASS Collaboration, V.Yu. Alexakhin et al., Phys. Lett. B 647 (2007) 8.

[5] COMPASS Collaboration, M.G. Alekseev et al., Phys. Lett. B 690 (2010) 466.

[6] COMPASS Collaboration, C. Adolph et al., The Spin Structure Function $g_{1}^{\mathrm{p}}$ of the proton and a test of the Bjorken Sum Rule, to be published in Phys. Lett. B, arXiv:1503.08935.

[7] E.R. Nocera et al., Nucl. Phys. B 887 (2014) 276.

[8] COMPASS Collaboration, C. Adolph et al., Phys. Rev. D 87 (2013) 052018.

[9] M. Stolarski, A new LO extraction of the gluon polarisation from DIS data, Proc. of the XXII. Int. Workshop on Deep Inelastic Scattering and Related Subjects, Warsaw (2014), PoS DIS2014 (2014) 211.

[10] COMPASS Collaboration, C. Adolph et al., Phys. Lett. B 718 (2013) 922.

[11] D. de Florian, R. Sassot, M. Stratmann and W. Vogelsang, Phys. Rev. Lett. 113 (2014) 012001.

[12] COMPASS Collaboration, M.G. Alekseev et al., Phys. Lett. B 680 (2009) 217, Phys. Lett. B 693 (2010) 227.

[13] E. Seder, $K^{ \pm}$multiplicities in semi-inclusive deep-inelastic Scattering from COMPASS, Proc. of the XXIII. Int. Workshop on Deep Inelastic scattering and Related Subjects, Dallas (2015), to be publisehd by PoS.

[14] M. Hirai, S. Kumano, Comut. Phys. Commun. 183 (2012) 1002.

[15] E. Leader, A.V. Sidorov and D. Stamenov, Importance of Semi-Inclusive DIS Processes in Determining Fragmentation Functions, 15th Workshop on High Energy Physics (DPSIN-13), Dubna, Russia (2013), arXiv:1312.5200.

[16] D. de Florian, R. Sassot and M. Stratmann, Phys. Rev. D 75 (2007) 114010.

[17] D. de Florian et al., Phys. Rev. D 91 (2015) 014045. 